Review Article

\title{
Motor and Nonmotor Symptoms of Parkinson's Disease: Antagonistic Pleiotropy Phenomena Derived from $\alpha$-Synuclein Evolvability?
}

\author{
Yoshiki Takamatsu, ${ }^{1}$ Masayo Fujita, ${ }^{1}$ Gilbert J. Ho ${ }^{D},{ }^{2}$ Ryoko Wada, ${ }^{1}$ Shuei Sugama, ${ }^{3}$ \\ Takato Takenouchi $\left(\mathbb{D},{ }^{4}\right.$ Masaaki Waragai, ${ }^{1}$ Eliezer Masliah, ${ }^{5}$ and Makoto Hashimoto ${ }^{1}$ \\ ${ }^{1}$ Tokyo Metropolitan Institute of Medical Science, 2-1-6 Kamikitazawa, Setagaya-ku, Tokyo, Japan \\ ${ }^{2}$ PCND Neuroscience Research Institute, Poway, CA, USA \\ ${ }^{3}$ Department of Physiology, Nippon Medical School, Tokyo, Japan \\ ${ }^{4}$ Institute of Agrobiological Sciences, National Agriculture and Food Research Organization, Tsukuba, Ibaraki, Japan \\ ${ }^{5}$ Department of Neuroscience, National Institute on Aging, Bethesda, MD 20892, USA \\ Correspondence should be addressed to Makoto Hashimoto; hashimoto-mk@igakuken.or.jp
}

Received 13 August 2018; Revised 1 October 2018; Accepted 18 October 2018; Published 22 November 2018

Guest Editor: Matteo Bologna

Copyright (c) 2018 Yoshiki Takamatsu et al. This is an open access article distributed under the Creative Commons Attribution License, which permits unrestricted use, distribution, and reproduction in any medium, provided the original work is properly cited.

\begin{abstract}
Lewy body diseases, such as Parkinson's disease (PD), dementia with Lewy bodies (DLB), and multiple system atrophy (MSA), are associated with a wide range of nonmotor symptoms (NMS), including cognitive impairment, depression and anxiety, sleep disorders, gastrointestinal symptoms, and autonomic failure. The reason why such diverse and disabling NMS have not been weeded out but have persisted across evolution is unknown. As such, one possibility would be that the NMS might be somehow beneficial during development and/or reproductive stages, a possibility consistent with our recent view as to the evolvability of amyloidogenic proteins (APs) such as $\alpha$-synuclein $(\alpha \mathrm{S})$ and amyloid- $\beta(\mathrm{A} \beta)$ in the brain. Based on the heterogeneity of protofibrillar AP forms in terms of structure and cytotoxicity, we recently proposed that APs might act as vehicles to deliver information regarding diverse internal and environmental stressors. Also, we defined evolvability to be an epigenetic phenomenon whereby APs are transgenerationally transmitted from parents to offspring to cope with future brain stressors in the offspring, likely benefitting the offspring. In this context, the main objective is to discuss whether NMS might be relevant to evolvability. According to this view, information regarding NMS may be transgenerationally transmitted by heterogeneous APs to offspring, preventing or attenuating the stresses related to such symptoms. On the other hand, NMS associated with Lewy body pathology might manifest through an aging-associated antagonistic pleiotropy mechanism. Given that NMS are not only specific to Lewy body diseases but also displayed in other disorders, including amyotrophic lateral sclerosis (ALS) and Huntington's disease (HD), these conditions might share common mechanisms related to evolvability. This might give insight into novel therapy strategies based on antagonistic pleiotropy rather than on individual NMS from which to develop diseasemodifying therapies.
\end{abstract}

\section{Introduction}

It is well established that synucleinopathies, including PD, DLB, and MSA, are characterized by a number of NMS, such as cognitive impairment, depression and anxiety, sleep difficulties, gastrointestinal disturbance, and autonomic failure. Because some NMS occur in the prodromal disease stages, NMS are both mechanistically and therapeutically important [1,2]. Recently, in this field, there has been great interest in better understanding NMS, a topic which has 
been prominently reviewed [3-9]. Nevertheless, the mechanisms which underlie NMS in neurodegenerative diseases remain obscure.

Accordingly, the main objective of this paper is to discuss how NMS might be involved in the pathogenesis of synucleinopathies and related disorders. Given that a variety of NMS often occur during the course of multiple neurodegenerative conditions, it is predicted that NMS might be triggered by multiple pathologic factors, including protein aggregation and inflammation. One possibility then would be that NMS might be passive phenomena as a result of neurodegeneration. Yet, an alternative and nonmutually exclusive possibility is that NMS might be a consequence of evolvability [10], whereby NMS information might be transgenerationally delivered to offspring encoded in APs, such as $\alpha \mathrm{S}$ and $\mathrm{A} \beta$, perhaps preventing the stresses relevant to NMS in offspring. On the other hand, NMS may manifest as symptoms of aging-associated neurodegenerative disease through an antagonistic pleiotropy mechanism in the parental brains. Finally, we propose that a better understanding of this hypothetical view would facilitate development of a therapy strategy against NMS in synucleinopathies.

\section{Motor and Nonmotor Symptoms in Synucleinopathies}

In $\mathrm{PD}$ and related synucleinopathies, treating motor signs and symptoms due to the degeneration of dopaminergic neurons in the substantia nigra has long been the focus of disease management. However, in recent years, because of increased clinical recognition and relevance to patient life quality, the nonmotor aspects of such disorders have attracted increasing interest. Clinically, NMS consists of four domains: neuropsychiatric (e.g., depression, anxiety, apathy, hallucinations, and dementia), autonomic (e.g., constipation, orthostatic hypotension, urinary changes, and sweating abnormalities), sleep (e.g., insomnia, sleep fragmentation, excessive daytime sleepiness, rapid eye movement, sleep disorder, and restless leg syndrome), and sensory dysfunction (e.g., pain and olfactory dysfunction) [11-13]. Such diversity of NMS may be consistent with the widespread distribution of $\alpha \mathrm{S}$ pathology in the gut $[14,15]$ as well as brainstem and neocortex in PD brain [16], in which multiple populations of aminergic neurons may be affected, including serotonergic and noradrenergic neurons. Thus, the classic Parkinsonian motor syndrome is now regarded as but one unitary symptom type among many disparate symptoms of the synucleinopathies.

\section{Are NMS Passive Phenomena?}

Overall, it would appear possible that similar to motor symptoms (MS), NMS might be passive phenomena in response to amyloid fibrils and inflammation during the progression of PD and other disorders, including ALS and HD [17-20]. Indeed, such a view is supported by the results of studies in animal models. For instance, we also observed that transgenic (Tg) mice expressing DLB-linked $\mathrm{P} 123 \mathrm{H} \beta$ synuclein $(\beta S)$ developed progressive neurodegeneration, as characterized by axonal swelling, astrogliosis, and behavioural abnormalities. Interestingly, expression of the memory abnormality ( $\sim 6$ months of age on water maze testing) was more prominent compared with the motor deficits ( 12 months of age on the rotarod treadmill test) [21] (Figures 1(a) and 1(b)). Furthermore, P123H $\beta \mathrm{S}$ mice exhibited depression-like behaviors as assessed by locomotor activity ( $\sim 6$ months) and the nest building test $(\sim 6$ months) [22] (Figures 1(c) and 1(d)). Collectively, this suggested that motor deficits were preceded by NMS, such as memory dysfunction and depression-like features. Similarly, hyperactivity and depression-like behaviors were observed in A53T $\alpha$ S Tg mice [23] and a tauopathy mouse model [24]. Since APs are constitutively expressed using artificial promoters, such as thy-1, prion promoter, and calmodulin kinase II $\alpha$, in Tg mice models of neurodegenerative diseases $[21,23,24]$, it is presumed that the accumulation of protofibrillar APs, including $\mathrm{P} 123 \mathrm{H} \beta \mathrm{S}, \alpha \mathrm{S}$, and tau, may interfere with signal transduction and transcription, eventually leading to the manifestation of neurobehavioral phenotypes such as depression.

There, however, would seem to be little evolutionary advantage for the passive association of NMS with neurodegenerative diseases in aging. Distinct from other organisms, humans are characterized by an extended postmenopausal senescence due to stable nutritional supply and an absence of predators [25]. Although nature remains biologically indifferent to the human condition during postreproductive time of life, a recent study suggests that the "grandmother effect" in humans may be evolutionarily beneficial because nursing of their first grandchild by a grandmother is beneficial to their daughter to encourage birth of a second grandchild [26]. From this perspective, if NMS are simply passive phenomena following neurodegeneration features, such as accumulation of toxic aggregates of APs and inflammation during aging, this would be evolutionarily not advantageous and might have been selected out in evolution.

\section{NMS as Active Phenomena Dependent on Evolvability and Antagonistic Pleiotropy}

One might wonder as to why NMS have not been eliminated through natural selection. Indeed, it was recently described that both MS and NMS were observed in 1-methyl-4phenyl-1, 2, 3, 6-tetrahydropyridine-treated marmosets, a nonhuman primate model [27]. Considering that NMS by themselves are rather consequences that are severely disabling for patients in aging and cannot transgenerationally be delivered to offspring, we predict that NMS might be linked to some physiologically beneficial effects during development and/or reproductive stages. Notably, such a view is reminiscent of the evolvability of APs such as $\alpha \mathrm{S}$ and $\mathrm{A} \beta$ in the brain [10]. Based on the heterogeneity of protofibrillar forms of APs in terms of structure and cytotoxicity, we proposed that APs might act as vehicles to deliver information regarding diverse biological stressors [10]. Mechanistically, we speculate that $\alpha \mathrm{S}$, a monomer of which is unstable due to its intrinsically disordered nature [28], might become more stable through oligomerization, 


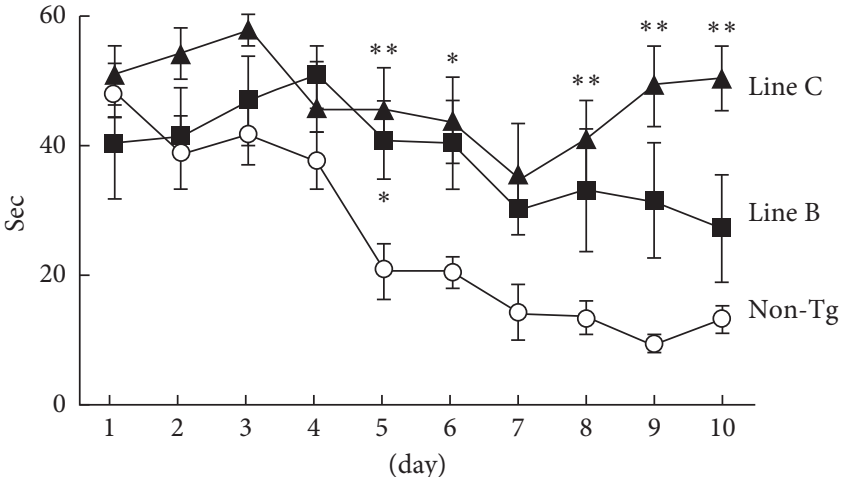

(a)

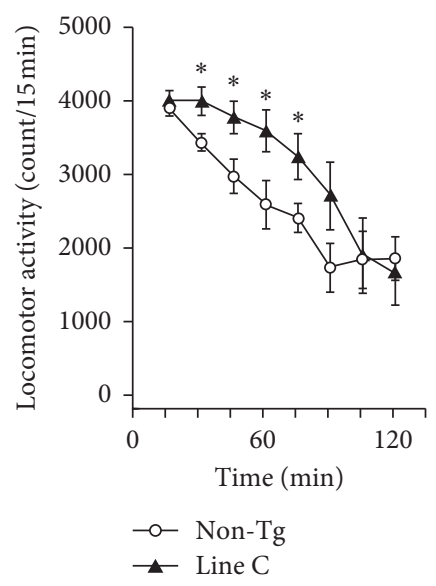

(c)

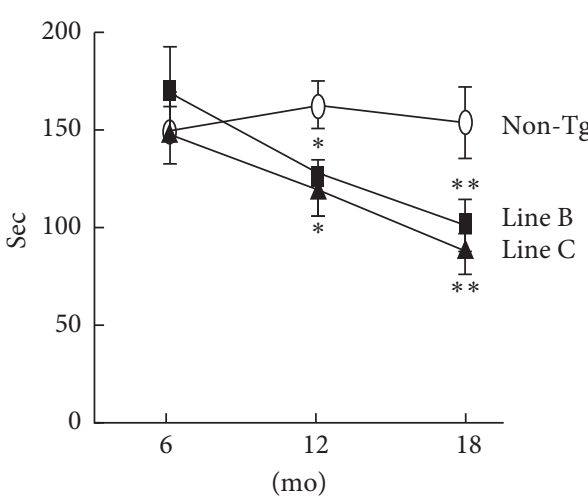

(b)

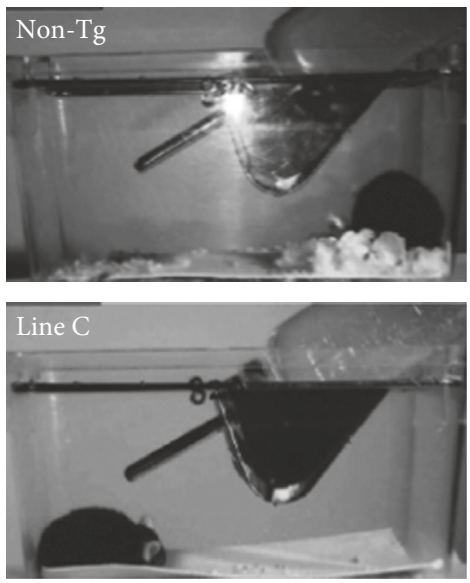

(d)

Figure 1: Altered behaviors observed in a DLB model mouse (a and b). Tg mice expressing DLB-linked P123H $\beta$ S were characterized by memory disorder ( $\sim 6$ month: by the water maze test) (a) and being more prominent than motor deficits ( 12 month: by the rotarod treadmill test) (b). See Reference [21] for the details. (c and d) The P123H $\beta$ S mice exhibited depression-like behaviors as assessed from the results of the locomotor activity (6 10 month) (c) and the nest building test (6 10 month) (d). See Reference [21] for the details. Reprinted with permission from References [21, 22].

leading to formation of diverse strains of protofibrils. Such stable $\alpha \mathrm{S}$ protofibrils may be feasible for transgenerational transmission to the offspring.

In this way, information regarding both MS and NMS might be integrated into the evolvability of $\alpha \mathrm{S}$ (Figure 2). Presuming that NMS-related information is transgenerationally transmitted to offspring through evolvability of $\alpha \mathrm{S}$, it would benefit offspring. Yet, on the other hand, $\alpha \mathrm{S}$ aggregates may also cause neurodegenerative disease and associated NMS through an antagonistic pleiotropy mechanism during aging. Thus, evolvability would be an epigenetic phenomenon in which APs transgenerationally transmit such information to offspring to cope with future stressors affecting the offspring's brain. It is predicted that NMS might be active phenomena related to evolvability.

\section{Modulation of NMS Evolvability by Other Factors}

Because $\alpha$ S pathology is promoted by other APs, such as $\mathrm{A} \beta$ [29] and tau [30], it is likely that evolvability of these molecules might also positively affect the evolvability of $\alpha \mathrm{S}$
(Figure 2). Furthermore, $\beta S$ is also of particular interest because the evolvability of $\alpha \mathrm{S}$ may be positively and negatively regulated by wild-type and mutant $\beta S$, respectively $[21,31]$. Similarly, since $\gamma$-synuclein $(\gamma S)$, the third member of the synuclein family of peptides [32,33], may be involved in the regulation of $\alpha \mathrm{S}$ evolvability because $\gamma \mathrm{S}$ is associated with neuritic pathology, such as in dystrophic neurites and spheroid structures, in the brains of sporadic cases of PD, $\mathrm{DLB}$, and neurodegeneration with brain iron accumulation type $1[34,35]$. Furthermore, it was shown that the formation of aggregates and deposits of $\gamma \mathrm{S}$ is facilitated after its oxidation at methionine 38 [36]. Collectively, it is possible that all synuclein family peptides might cooperate in NMSrelated $\alpha \mathrm{S}$ evolvability.

Moreover, aggregation of $\alpha \mathrm{S}$ was also shown to be influenced by apolipoprotein E (apoE), a major Alzheimer's disease (AD) risk factor, with apoE4 having the most robust stimulatory effect compared with other isoforms (E2 and E3). Since apoE4 binds to $A \beta$ and promotes fibrillization, we previously suggested that evolvability of $\mathrm{A} \beta$ might be enhanced by apoE4 [25]. Similarly, other apolipoproteins, such as ApoJ, and ApoA1, might also associate with $\alpha \mathrm{S}$ to modify 


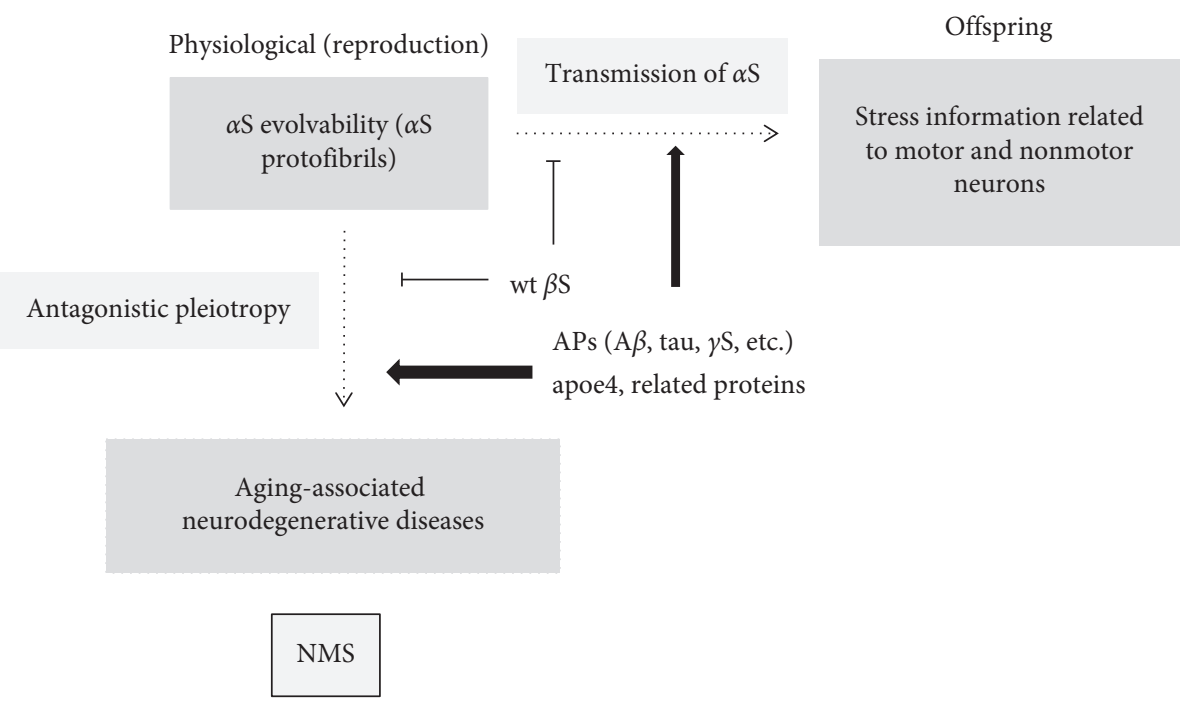

FIgURE 2: Schematics of the motor and nonmotor symptoms in neurodegenerative diseases and amyloid evolvability in the human brain. Hypothetically, stress information derived from both motor and nonmotor neurons might be integrated into diverse structures of aS protofibril strains that are transgenerationally transmitted to offspring during reproduction as a physiological phenomenon. On the other hand, the $\alpha \mathrm{S}$ protofibrils may manifest as neurodegenerative disease associated with NMS through an antagonistic pleiotropy mechanism during the postreproductive senescent period. Both processes are stimulated by various proteins, including $\mathrm{A} \beta$, tau, $\gamma \mathrm{S}$, and apoE, but are suppressed by wild-type $\beta$ S.

evolvability [37, 38]. Notably, the importance of membrane lipids, such as raft, in $\alpha$-synucleinopathies has been previously described [39]. Thus, it is tempting to speculate that the pathological role of membranous functions in $\alpha$-synucleinopathies in aging might reflect the regulation of $\alpha$ S evolvability by the membrane in development/reproduction.

In addition, there has been increasing interest in transgenerational epigenetic inheritance in which various epigenetic factors like DNA methylation, histone modifications, and regulatory RNAs have been described [40]. Therefore, it is possible that some of these epigenetic factors are involved in regulating $\alpha \mathrm{S}$ evolvability (Figure 2).

\section{Therapeutic Implication}

Notably, some NMS such as cognitive and neuropsychiatric features, [1] as well as constipation and other gastrointestinal symptoms [2], often are expressed in the prodromal disease stage of neurodegeneration. Since recent studies suggest that disease-modifying therapy (DMT) for neurodegenerative diseases should be initiated at earlier stages, NMS may be important from both the mechanistic and therapeutic standpoints.

As discussed, NMS might be either passive phenomena during the course of neurodegeneration or an active phenomena derived from evolvability through antagonistic pleiotropy. In the former case, neuropathogenic factors, such as fibrils and inflammation, are presumed to be situated upstream of NMS. Although therapeutic strategies are thought to target those neuropathogenic factors, no DMT has thus proven effective in relieving NMS. Alternatively, each nonmotor symptom might be individually targeted. For instance, dysfunction of hypothalamic-pituitary-adrenal axis (HPA), a central regulatory system underlying stressors [41], has been implicated in contributing to depressed mood and anxiety, in patients with depression [42]. In this context, it was shown that deletion of corticotropinreleasing factor receptor type 1 (CRFR1) mitigated the amyloid- $\beta$ pathology in a mouse model of $\mathrm{AD}$, lending support to the notion that suppressing the HPA axis through CRFR1 antagonism may be an effective therapeutic strategy against AD [43]. Given that CRFR1 in the brain is involved in the regulation of endocrine, behavioural, autonomic, and visceral in response to stress [44], the suppression of CRFR1 signaling might also be effective for other neurodegenerative diseases with NMS conditions. Also, pharmacological approaches, such as NMDA antagonists and dopamine agonists might be effective for some NMS such as depression $[45,46]$.

Yet, if the alternate explanation is the case, more unconventional therapeutic strategies might be employed. For instance, in addition to targeting neuropathogenic factors, such as fibrils and inflammation, disease-modifying strategies would focus on antagonistic pleiotropy rather than on the individual NMS. Currently, the mechanism underlying antagonistic pleiotropy is unclear. In this regard, however, it is noteworthy that a recent study revealed pleiotropic associations of allelic variants in a 2q22 region with risks of major human diseases, such as vascular disease, cancer, and neurodegenerative disease, and mortality [47], suggesting a possibility that the serine/threonine TGF $\beta /$ activin receptor-signaling pathways might be involved in the regulation of antagonistic pleiotropy. In support of this view, importance of the serine129 with phosphorylates $\alpha \mathrm{S}$ has been well characterized in PD [48]. In particular, accumulation of $\alpha$ S serine 129 phosphorylation in Lewy bodies is a hallmark of the pathogenesis in PD [49]. The similar is the case of tau in $\mathrm{AD}$ although involvement of both 
serine/threonine kinases and tyrosine kinase has been described $[50,51]$. If this view is the case, modification of the TGF $\beta /$ activin receptor-signaling pathways could be therapeutically effective for the entire symptoms, including both MS and NMS in neurodegenerative diseases and perhaps other aging-associated chronic diseases. Further investigations are warranted to test this intriguing possibility.

\section{Conclusions}

Although increasingly clear that NMS are important early biomarkers as well as targets for disease-modifying therapy for synucleinopathies, such as PD, DLB, and MSA, the mechanisms by which NMS are involved in the pathogenesis of the disease have not been fully understood. We hypothesized that stress information derived from both MSand NMS-relevant neurons might be integrated into the diverse structures of $\alpha \mathrm{S}$ protofibrils and are transgenerationally transmitted, which is probably beneficial to ward against forthcoming stressors in offspring, i.e., evolvability.

However, in parental brain, $\alpha \mathrm{S}$ protofibrils might manifest later in life associated with aging-associated neurodegenerative disorders through the antagonistic pleiotropy mechanism. Therefore, our theory implies that NMS, because they are derived from the physiological phenomenon of evolvability, are not selected by evolution. It further introduces a new framework that antagonistic pleiotropy might be a valid therapeutic target for disease-associated NMS.

Although the concepts of amyloid evolvability and the antagonistic pleiotropy phenomena derived from amyloidlike proteins in neurodegenerative diseases are intriguing, such a theory requires further experimental validations and at present is far from explaining the complex pathophysiology of NMS in PD. Thus, further investigations are definitely warranted to demonstrate our hypothesis.

\section{Conflicts of Interest}

The authors declare that they have no conflicts of interest.

\section{Authors' Contributions}

MH conceived the study, and MH, YT, and GH wrote the paper. All authors have read and approved the manuscript.

\section{Acknowledgments}

We are grateful for the continuous encouragement of Drs. Kaori Hashimoto (Tokyo Metropolitan Institute of Medical Science) and Maria del Carmen Ruiz de la Cruz (University of Chicago).

\section{References}

[1] S. J. Getz and B. Levin, "Cognitive and neuropsychiatric features of early Parkinson's disease," Archives of Clinical Neuropsychology, vol. 32, no. 7, pp. 769-785, 2017.
[2] K. Knudsen, M. Szwebs, A. K. Hansen, and P. Borghammer, "Gastric emptying in Parkinson's disease-a mini-review," Parkinsonism \& Related Disorders, vol. 55, pp. 18-25, 2018.

[3] W. Poewe, "Non-motor symptoms in Parkinson's disease," European Journal of Neurology, vol. 15, no. 1, pp. 14-20, 2008.

[4] A. M. Bonnet, M. F. Jutras, V. Czernecki, J. C. Corvol, and M. Vidailhet, "Nonmotor symptoms in Parkinson's disease in 2012: relevant clinical aspects," Parkinson's Disease, vol. 2012, article 198316, 15 pages, 2012.

[5] D. H. Ffytche, B. Creese, M. Politis et al., "The psychosis spectrum in Parkinson disease," Nature Reviews Neurology, vol. 13, no. 2, pp. 81-95, 2017.

[6] D. Aarsland, B. Creese, M. Politis et al., "Cognitive decline in Parkinson disease," Nature Reviews Neurology, vol. 13, no. 4, pp. 217-231, 2017.

[7] A. A. Poirier, B. Aube, M. Cote, N. Morin, T. Di Paolo, and D. Soulet, "Gastrointestinal dysfunctions in Parkinson's disease: symptoms and treatments," Parkinson's Disease, vol. 2016, Article ID 6762528, 23 pages, 2016.

[8] R. S. Weil, A. E. Schrag, J. D. Warren, S. J. Crutch, A. J. Lees, and H. R. Morris, "Visual dysfunction in Parkinson's disease," Brain, vol. 139, no. 11, pp. 2827-2843, 2016.

[9] P. Zis, R. Erro, C. C. Walton, A. Sauerbier, and K. R. Chaudhuri, "The range and nature of non-motor symptoms in drug-naive Parkinson's disease patients: a state-of-the-art systematic review," NPJ Parkinson's Disease, vol. 1, no. 1, article 15013, 2015.

[10] M. Hashimoto, G. Ho, S. Sugama et al., "Evolvability of amyloidogenic proteins in human brain," Journal of Alzheimer's Disease, vol. 62, no. 1, pp. 73-83, 2018.

[11] N. Modugno, F. Lena, F. Di Biasio, G. Cerrone, S. Ruggieri, and F. Fornai, "A clinical overview of non-motor symptoms in Parkinson's disease," Archives Italiennes de Biologie, vol. 151, no. 4, pp. 148-168, 2013.

[12] C. Pellicano, F. Assogna, L. Cravello et al., "Neuropsychiatric and cognitive symptoms and body side of onset of parkinsonism in unmedicated Parkinson's disease patients," Parkinsonism \& Related Disorders, vol. 21, no. 9, pp. 1096-1100, 2015.

[13] S. M. van Rooden, M. Visser, D. Verbaan, J. Marinus, and J. J. van Hilten, "Patterns of motor and non-motor features in Parkinson's disease," Journal of Neurology, Neurosurgery \& Psychiatry, vol. 80, no. 8, pp. 846-850, 2009.

[14] K. Shannon and P. Vanden Berghe, "The enteric nervous system in PD: gateway, bystander victim, or source of solutions," Cell and Tissue Research, vol. 373, no. 1, pp. 313-326, 2018.

[15] E. Stolzenberg, D. Berry, D. Yang et al., "A role for neuronal alpha-synuclein in gastrointestinal immunity," Journal of Innate Immunity, vol. 9, no. 5, pp. 456-463, 2017.

[16] C. H. Hawkes, K. Del Tredici, and H. Braak, "Parkinson's disease: a dual-hit hypothesis," Neuropathology and Applied Neurobiology, vol. 33, no. 6, pp. 599-614, 2007.

[17] N. Stefanova, K. Seppi, C. Scherfler, Z. Puschban, and G. K. Wenning, "Depression in alpha-synucleinopathies: prevalence, pathophysiology and treatment," in Advances in Research on Neurodegeneration, P. Riederer, Ed., pp. 335-343, Springer, Vienna, Austria, 2000.

[18] D. W. Dickson, H. Fujishiro, C. Orr et al., "Neuropathology of non-motor features of Parkinson disease," Parkinsonism \& Related Disorders, vol. 15, no. 3, pp. S1-S5, 2009.

[19] A. M. Blokhuis, E. J. Groen, M. Koppers, L. H. van den Berg, and R. J. Pasterkamp, "Protein aggregation in amyotrophic 
lateral sclerosis," Acta Neuropathologica, vol. 125, no. 6, pp. 777-794, 2013.

[20] F. Cardoso, "Nonmotor symptoms in Huntington disease," International Review of Neurobiology, vol. 134, pp. 1397-1408, 2017.

[21] M. Fujita, S. Sugama, K. Sekiyama et al., "A beta-synuclein mutation linked to dementia produces neurodegeneration when expressed in mouse brain," Nature Communications, vol. 1, no. 8, p. 110, 2010.

[22] M. Fujita, Y. Hagino, Y. Takamatsu et al., "Early manifestation of depressive-like behavior in transgenic mice that express dementia with Lewy body-linked mutant $\beta$-synuclein," Neuropsychopharmacology Reports, vol. 38, no. 2, pp. 95-97, 2018.

[23] K. L. Paumier, S. J. Sukoff Rizzo, Z. Berger et al., "Behavioral characterization of A53T mice reveals early and late stage deficits related to Parkinson's disease," PLoS One, vol. 8, no. 8, Article ID e70274, 2013.

[24] P. Jul, C. Volbracht, I. E. de Jong, L. Helboe, A. B. Elvang, and J. T. Pedersen, "Hyperactivity with agitative-like behavior in a mouse tauopathy model," Journal of Alzheimer's Disease, vol. 49, no. 3, pp. 783-795, 2016.

[25] M. Hashimoto, G. Ho, Y. Takamatsu et al., "Evolvability and neurodegenerative disease: antagonistic pleiotropy phenomena derived from amyloid aggregates," Journal of Parkinson's Disease, vol. 8, no. 3, pp. 405-408, 2018.

[26] K. Hawkes, "Human longevity: the grandmother effect," Nature, vol. 428, no. 6979, pp. 128-129, 2004.

[27] G. R. Choudhury and M. M. Daadi, "Charting the onset of Parkinson-like motor and non-motor symptoms in nonhuman primate model of Parkinson's disease," PLoS One, vol. 13, no. 8, Article ID e0202770, 2018.

[28] T. Bartels, L. S. Ahlstrom, A. Leftin et al., "The N-terminus of the intrinsically disordered protein alpha-synuclein triggers membrane binding and helix folding," Biophysical Journal, vol. 99, no. 7, pp. 2116-2124, 2010.

[29] E. Masliah, E. Rockenstein, I. Veinbergs et al., "Beta-amyloid peptides enhance alpha-synuclein accumulation and neuronal deficits in a transgenic mouse model linking Alzheimer's disease and Parkinson's disease," Proceedings of the National Academy of Sciences, vol. 98, no. 21, pp. 12245-12250, 2001.

[30] B. I. Giasson, M. S. Forman, M. Higuchi et al., "Initiation and synergistic fibrillization of tau and alpha-synuclein," Science, vol. 300, no. 5619, pp. 636-640, 2003.

[31] M. Hashimoto, E. Rockenstein, M. Mante, M. Mallory, and E. Masliah, "Beta-Synuclein inhibits alpha-synuclein aggregation: a possible role as an anti-parkinsonian factor," Neuron, vol. 32, no. 2, pp. 213-223, 2001.

[32] J. M. George, “The synucleins," Genome Biology, vol. 3, no. 1, p. $3002.1,2002$.

[33] A. Surguchov, "Intracellular dynamics of synucleins: "here, there and everywhere"," International Review of Cell and Molecular Biology, vol. 320, pp. 103-169, 2015.

[34] J. E. Galvin, K. Uryu, V. M. Lee, and J. Q. Trojanowski, “Axon pathology in Parkinson's disease and Lewy body dementia hippocampus contains alpha-, beta-, and gamma-synuclein," Proceedings of the National Academy of Sciences, vol. 96, no. 23, pp. 13450-13455, 1999.

[35] J. E. Galvin, B. Giasson, H. I. Hurtig, V. M. Lee, and J. Q. Trojanowski, "Neurodegeneration with brain iron accumulation, type 1 is characterized by alpha-, beta-, and gamma-synuclein neuropathology," American Journal of Pathology, vol. 157, no. 2, pp. 361-368, 2000.
[36] I. Surgucheva, K. L. Newell, J. Burns, and A. Surguchov, "New alpha- and gamma-synuclein immunopathological lesions in human brain," Acta Neuropathologica Communications, vol. 2, no. 1, p. 132, 2014.

[37] M. Calero, A. Rostagno, and J. Ghiso, "Search for amyloidbinding proteins by affinity chromatography," Methods in Molecular Biology, vol. 849, pp. 213-223, 2012.

[38] F. N. Emamzadeh and D. Allsop, "Alpha-synuclein interacts with lipoproteins in plasma," Journal of Molecular Neuroscience, vol. 63, no. 2, pp. 165-172, 2017.

[39] S. I. Kubo, "Membrane lipids as therapeutic targets for Parkinson's disease: a possible link between Lewy pathology and membrane lipids," Expert Opinion on Therapeutic Targets, vol. 20, no. 11, pp. 1301-1310, 2016.

[40] A. Sharma, "Transgenerational epigenetics: integrating soma to germline communication with gametic inheritance," Mechanisms of Ageing and Development, vol. 163, pp. 15-22, 2017.

[41] S. M. Smith and W. W. Vale, "The role of the hypothalamicpituitary-adrenal axis in neuroendocrine responses to stress," Dialogues in Clinical Neuroscience, vol. 8, no. 4, pp. 383-395, 2006.

[42] E. A. Young, J. L. Abelson, and O. G. Cameron, "Effect of comorbid anxiety disorders on the hypothalamic-pituitaryadrenal axis response to a social stressor in major depression," Biological Psychiatry, vol. 56, no. 2, pp. 113-120, 2004.

[43] C. Zhang, C. C. Kuo, S. H. Moghadam, L. Monte, K. C. Rice, and R. A. Rissman, "Corticotropin-releasing factor receptor-1 antagonism reduces oxidative damage in an alzheimer's disease transgenic mouse model," Journal of Alzheimer's Disease, vol. 45, no. 2, pp. 639-650, 2015.

[44] J. Czimmer and Y. Tache, "Peripheral corticotropin releasing factor signaling inhibits gastric emptying: mechanisms of action and role in stress-related gastric alterations of motor function," Current Pharmaceutical Design, vol. 23, no. 27, pp. 4042-4047, 2017.

[45] B. Vanle, W. Olcott, J. Jimenez, L. Bashmi, I. Danovitch, and W. W. IsHak, "NMDA antagonists for treating the non-motor symptoms in Parkinson's disease," Translational Psychiatry, vol. 8, no. 1, p. 117, 2018.

[46] E. Dalle and M. V. Mabandla, "Early life stress, depression and Parkinson's disease: a new approach," Molecular Brain, vol. 11, no. 1, p. 18, 2018.

[47] A. M. Kulminski, L. He, I. Culminskaya et al., "Pleiotropic associations of allelic variants in a $2 \mathrm{q} 22$ region with risks of major human diseases and mortality," PLOS Genetics, vol. 12, no. 11, article e1006314, 2016.

[48] T. Iwatsubo, "Aggregation of alpha-synuclein in the pathogenesis of Parkinson's disease," Journal of Neurology, vol. 250, no. 3, p. 1, 2003.

[49] V. M. Lee and J. Q. Trojanowski, “Mechanisms of Parkinson's disease linked to pathological alpha-synuclein: new targets for drug discovery," Neuron, vol. 52, no. 1, pp. 33-38, 2006.

[50] J. Z. Wang, Y. Y. Xia, I. Grundke-Iqbal, and K. Iqbal, "Abnormal hyperphosphorylation of tau: sites, regulation, and molecular mechanism of neurofibrillary degeneration," Journal of Alzheimer's Disease, vol. 33, no. 1, pp. S123-139, 2013.

[51] T. Lebouvier, T. M. Scales, R. Williamson et al., "The microtubule-associated protein tau is also phosphorylated on tyrosine," Journal of Alzheimer's Disease, vol. 18, no. 1, pp. 1-9, 2009. 


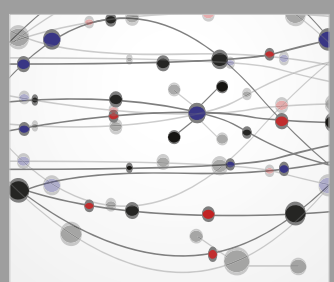

The Scientific World Journal
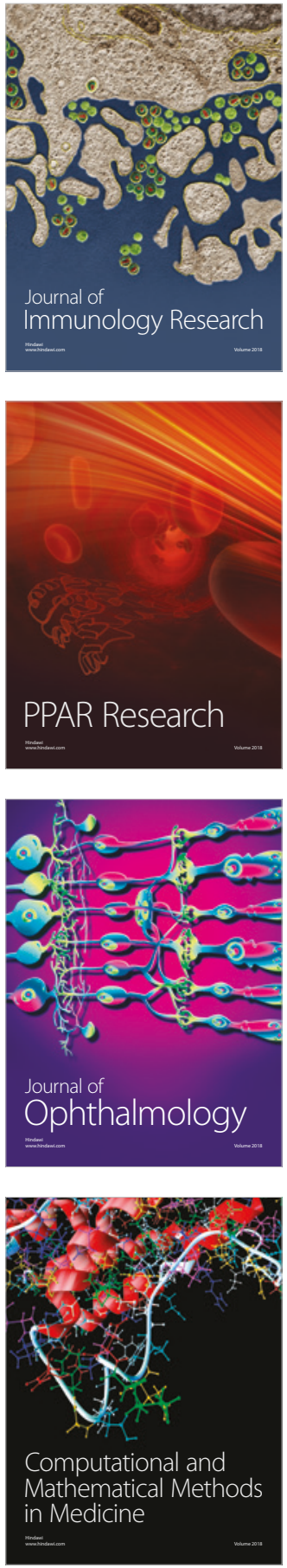

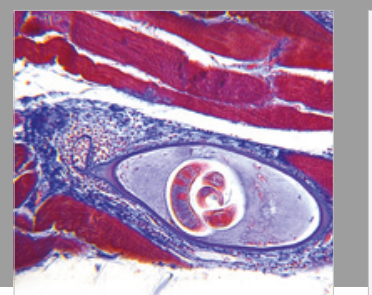

Gastroenterology Research and Practice

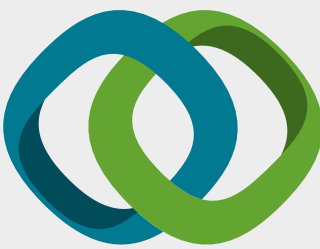

\section{Hindawi}

Submit your manuscripts at

www.hindawi.com
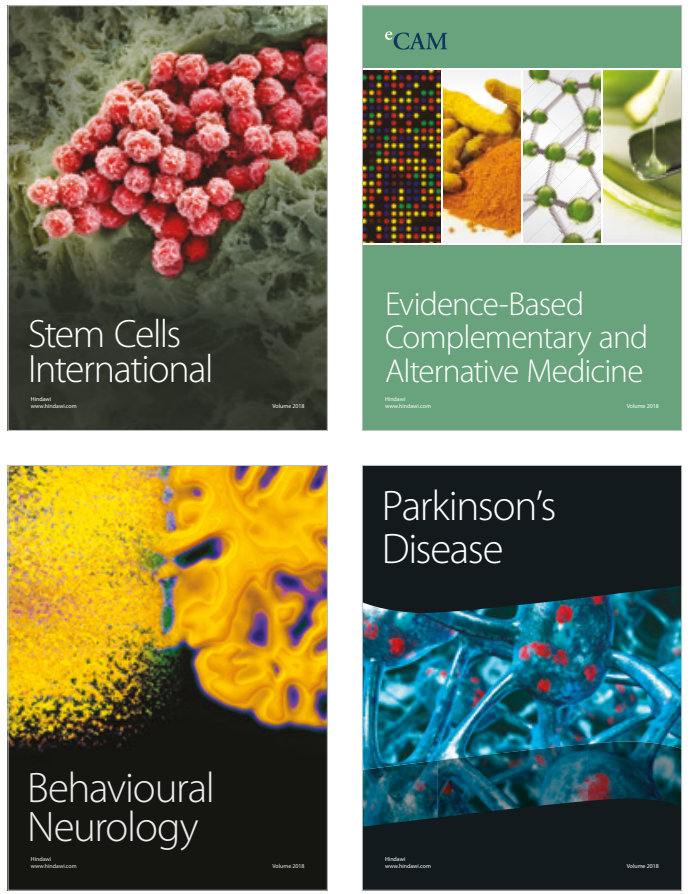

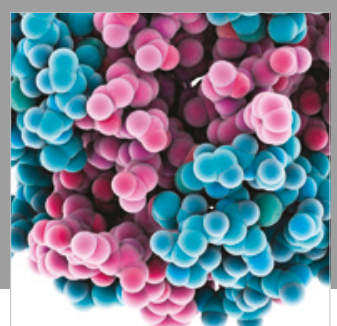

ournal of

Diabetes Research

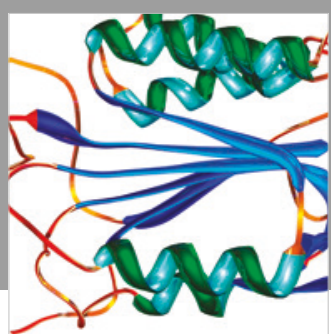

Disease Markers
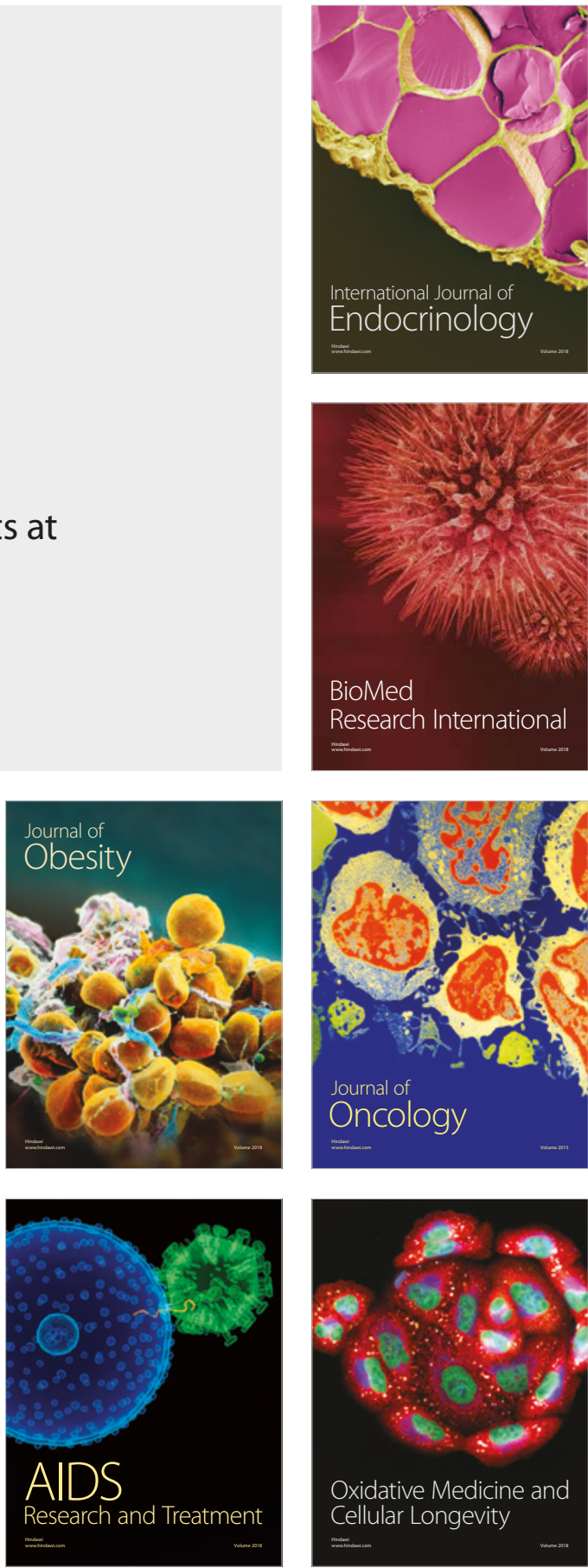